\title{
On One Model of Multichannel Queuing System with Unreliable Repairable Servers and Input Memory
}

\author{
Ramaz R. Shamugia ${ }^{1,2}$ \\ ${ }^{1}$ Research Department of Radiophysics and Electronic Systems Modeling, llia Vekua Sukhumi Institute of \\ Physics and Technology, Tbilisi, Georgia \\ ${ }^{2}$ Faculty of Mathematics and Computer Sciences, Sokhumi State University, Tbilisi, Georgia \\ Email: $\underline{\text { rmz.shamugia@gmail.com }}$
}

Received 19 January 2014; revised 25 February 2014; accepted 20 March 2014

Copyright (c) 2014 by author and Scientific Research Publishing Inc.

This work is licensed under the Creative Commons Attribution International License (CC BY). http://creativecommons.org/licenses/by/4.0/

(c)

\section{Abstract}

The work deals with the development of analytical model of multichannel technical queuing system with unreliable servers and input memory where server failure flows and incoming request flows comply with Poissonian laws, while the flows of failed facilities repairs and flows of incoming requests comply with exponential laws of probability distribution. Random process of system change-over is a Markovian process with continuous time and discrete states. Relations binding basic parameters and output characteristics of the system indicated are obtained as probabilities of system staying in the given moment in one of the possible states. The proposed model is the most generalized compared to some models known in literature which could be considered as special cases of the considered model.

\section{Keywords}

Multichannel Queuing Systems, QS with Unreliable Facilities, QS with Repairable Facilities, Servers with Failures, QS with Queues

\section{Introduction}

The assurance of high dependability is one of the main problems in development of effective systems of production automation and data processing, based on computer technique hardware and software.

At present the dependability of separate components making such systems does not satisfy continuously increasing demands. 
Aggravation of dependability problems is also furthered with continuous growth of system complexity (dimensionality), conditioned with the considerable widening of current tasks and steady increase of the responsibilities for the entrusted functions.

Alongside with the growth of demands for dependability the growth for the demands to system capacity conditioned with the necessity of solution of more complicated problems is also observed. One of the means of achieving high capacity of solution of great problems is their parallelization between homogeneous service facilities making the system.

Simultaneous support of high factors of dependability and capacity may be achieved in multichannel systems consisting of facilities homogeneous in dependability and capacity where calls for service orders incoming in random order are supported.

Considering the availability or possibility of introduction into such systems of structural as well as time redundancies, the necessity of development of theoretical methods (models) of prediction of their technical states in time and of optimization of resources input for the maintenance of dependability and capacity level becomes evident.

The availability of such methods enables to estimate factors of the existing systems and to determine the demands presented to designed systems and their subsystems.

The specificity of the processes of above described systems functioning in real time systems enables to consider them as multichannel technical queuing systems with unreliable facilities, serving random flows of random length tasks.

The complexity of the structure of such systems, modular approach to their construction, wide parallelism of facilities operation, stochastic character of fail and repair flows of the device, and also of the moments of tasks arrival for execution and the lengths of their maintenance provide the possibility of using analytical methods of dependability theory (DT) and queuing theory (QT) for modeling of random processes happening in the considered systems.

Mathematical model developed here allows to estimate the effect of such parameters on the chosen factors of the efficiency of functioning of such parameters of the considered technical QS as are the request flow parameter and parameters of facility dependability and service discipline.

The urgency of the development of the model is determined with the necessity to create theoretical bases and practical recommendations for the solution of significantly important scientific-technical problem — problem of assurance of high efficiency of failure proof and high capacity control and data processing means created on the basis of such systems and information processing and also economy of material and labor resources necessary for their design and implementation.

In References [1]-[11] are developed and investigated analytical models of technical QS in various statements differing from each other in structural parameters of the considered systems such as the quantity of service facilities, memory availability, reliable parameters of devices and also parameters of random call flows and their service. Unlike of the mentioned references the proposed work develops the most generalized model which in the form of concrete particular cases will contain some results known in literature.

\section{Purpose}

Proceeding from the above said the purpose of our work is to develop research model and probability estimation of the factors of efficiency of real time fail-proof and high-capacity systems functioning having equipment and time resources for maintenance of dependability and efficiency level and functioning in conditions of the effect of random factors-refusals, repairs, and random flow of random length tasks and random time of their service.

The proposed model is developed with consideration of the following assumptions:

- Transitions of the system between different states is realized under shared effect of several random processes - processes of incoming and service of demands, as well as processes of refusals and repairs of service facilities:

- Technical queuing system consists of $n$ identical service facilities;

- Service facilities are subjected to hard failures which obey Poisson law of probability distribution with parameter $\alpha$;

- Random process of failed facilities repair is described with exponential law of probability distribution with parameter $\mu$; 
- Poisson flow of requests incomes into the system for service the intensity of which equals $\lambda$;

- Incoming requests are served by exponential law with parameter $\beta$;

- Input memory is provided in the system for those requests which enter during time interval when all $i$ operable devices are busy serving the earlier entered requests;

- Queue length is equal to $k-l$ where $k$ is general quantity of requests being in the system in the considered time moment, and $l$ is the quantity of requests served at the given time moment $(l=\overline{0, i})$, where $l$ is the quantity of serving facilities operable by that moment.

For description of the process of system functioning in time introduce special probability functions $R_{i, n-i}^{l, k-l}(t)$ and $P_{i, n-i}^{l, k-l}(t)$ describing the process of transition of the system into different states, in correspondence with the causes conditioning these transitions-refusals and restoration of serving devices or income and service of requests.

\section{Introduction of Special Functions}

The introduced functions are determined as follows:

$R_{i, n-i}^{l, k-l}(t)$ is the probability of event when in time $t$ the system will be in state with $i$ operable and $n-i$ inoperable devices and, at the same time, general quantity of requests being in the system will equal $k$, from which the quantity being in service of requests will equal $l$, and the quantity being in queue will be $(k-l)$. The given probability describes the process of changing of system states in case of fixed values of upper indices, i.e. of factors of request incoming and serving $-l$ and $(k-l)$.

$P_{i, n-i}^{l, k-l}(t)$ is the probability of event when in time $t$ the system will be in the state with $i$ operable and $n-i$ inoperable devices and, at the same time, general quantity of requests being in the system will equal $k$, from which the quantity being in service of requests will equal $l$, and the quantity being in queue will be $(k-l)$. The given probability describes the process of changing of system states in case of fixed values of lower indices, i.e. dependability of system indices-failures and repairs of serving devices $l$ and $n-l$.

$H_{i, n-i}^{l, k-l}(t)$ in the probability of event when in time $t$ the system will be in the state with $i$ operable and $n-i$ inoperable devices and, at the same time, general quantity of requests being in the system will equal $k$, from which the quantity being in service of requests will equal $l$, and the quantity being in queue will be $(k-l)$. The given probability describes the process of changing of system states at continuously changing upper and lower $i$, $l, k, n-i$ functioning process.

$$
H_{i, n-i}^{l, k-l}(t)=R_{i, n-i}^{l, k-l}(t) * P_{i, n-i}^{l, k-l}(t) ; \quad k=\overline{0, \infty}, \quad n=\overline{0, \infty}, i=\overline{0, n-1}, \quad l=\overline{0, k-1}
$$

Rate fixing condition has the following form:

$$
\sum_{i=0}^{i=n-1} \sum_{l=0}^{l=k-1} H_{i, n-i}^{l, k-l}=1
$$

In order to determine intensities of system transitions at fixed values of upper indices $l$ and $k-l$, the following designations are introduced:

$a_{i, n-i}^{l, k-l}=\{i \alpha+(i+1) \alpha+(n-i) \mu+[n-(l-1)] \mu\}$ is the intensity of system transition being in moment $t$ in state $S_{i, n-i}^{l, k-l}(t)$ into one of the possible states, $S_{i+1, n-(i+1)}^{l, k-l}, S_{i, n-1}^{l-1, k-(l-1)}$ or $S_{i, n-1}^{l+1, k-(l+1)}$ caused with refusal of restoration of serving device;

$a_{i-1, n-(i-1)}^{l, k-l}=[n-(i-1)] \mu$ is the intensity of system transition being in moment $t$ in state $S_{i-1, n-(i-1)}^{l, k-l}$, into neighbouring state $S_{i, n-i}^{l, k-l}(t)$ caused with the restoration of service device;

$a_{i+1, n-(i+1)}^{l, k-l}=(i+1) \alpha$ is the intensity of system transition being in moment $t$ in state $S_{i+1, n-(i+1)}^{l, k-1}$ into neighbouring state $S_{i, n-i}^{l, k-l}(t)$ caused with the refusal of service device;

In order to determine intensities of system transitions at fixed values of lower indices $l$ and $n-i$, the following designations are introduced:

$b_{i, n-i}^{l, k-l}=\{l \beta+[k-(l-1) \lambda]-(k-l) \lambda+(l+1) \beta\}$ is the intensity of system transition being in moment $t$ in state $S_{i, n-i}^{l, k-l}(t)$ into one of the possible states $S_{i, n-1}^{l-1, k-(l-1)}$ or $S_{i, n-1}^{l+1, k-(l+1)}$ caused with income and service of regular request; 
$b_{i, n-i}^{l-1, k-(l-1)}=[k-(l-1)] \lambda$ is the intensity of system transition being in moment $t$ in state $S_{i, n-i}^{l-1, k-(l-1)}$ into neighbouring state $S_{i, n-i}^{l, k-l}$ caused with income of regular request;

$b_{i, n-i}^{l+1, k-(l+1)}=(l+1) \beta$ is the intensity of system transition being in moment $t$ in state $S_{i, n-i}^{l+1, k-(l+1)}$ into neighbouring state $S_{i, n-i}^{l, k-l}$ caused with serving of regular request;

Considering the possible changes of the system in infinitely small time interval $(t, t+\Delta t)$, with the help of above introduced probabilities, on the basis of probability reasoning, the following system of difference equations can be written describing the process of system functioning in time in assumption of fixed values of upper indices $l$ and $k$ :

$$
\begin{aligned}
R_{0, n}^{l, k-l}(t+\Delta t)= & \left(1-a_{0, n}^{l, k-l} \Delta t\right) R_{0, n}^{l, k-l}+a_{1, n-1}^{l, k-l} R_{1, n-1}^{l, k-l}(t) \Delta t+Q_{0, n-1}^{l, k-l}(t) \Delta t+o(\Delta t) ; \text { when } i=0 ; \\
R_{i, n-i}^{l, k-l}(t+\Delta t)= & \left(1-a_{i, n-i}^{l, k-l} \Delta t\right) R_{i, n-i}^{l, k-l}(t)+a_{i-1, n-(i-1)}^{l, k-l} R_{i-1, n-(i-1)}^{l, k-l}(t) \Delta t+a_{i+1, n-(i+1)}^{l, k-l} R_{i+1, n-(i+1)}^{l, k-l}(t) \Delta t \\
& +Q_{i, n-1}^{l, k-l}(t) \Delta t+o(\Delta t), \quad \text { when } 1 \leq i \leq n ;
\end{aligned}
$$

where

$$
Q_{i, n-1}^{l, k-l}(t) \Delta t=b_{i, n-i}^{l-1, k-(l-1)} P_{i, n-1}^{l-1, k-(l-1)}(t) \Delta t+b_{i, n-i}^{l+1, k-(l+1)} P_{i, n-1}^{l+1, k-(l+1)}(t) \Delta t
$$

Summands entering in the right part of Equality (3) should be obtained by solution of the following system of difference equations which describes the process of functioning of the considered system in assumption of fixed values of lower indices $i$ and $n-i$ :

$$
\begin{gathered}
P_{i, n-i}^{0, k}(t+\Delta t)=\left(1-b_{i, n-i}^{0, k} \Delta t\right) P_{i, n-i}^{0, k}(t)+b_{i, n-i}^{1, k-1} P_{i, n-1}^{1, k-1}(t) \Delta t+o(\Delta t), \text { when } l=0 ; \\
P_{i, n-i}^{l, k-l}(t+\Delta t)=\left(1-b_{i, n-i}^{l, k-l} \Delta t\right) P_{i, n-i}^{l, k-l}(t)+b_{i, n-i}^{l-1, k-(l-1)} P_{i, n-1}^{l-1, k-(l-1)}(t) \Delta t \\
+b_{i, n-i}^{l+1, k-(l+1)} P_{i, n-1}^{l+1, k-(l+1)}(t) \Delta t+o(\Delta t), \quad \text { when } 1 \leq l \leq k ;
\end{gathered}
$$

Below we define sense of the notion of probabilities in Equations (1), (2) and (3):

$R_{i, n-i}^{l, k-l}(t+\Delta t)$ is the probability of the event when the system being in time moment $t$ in one of the possible states $S_{i, n-i}^{l, k-l}, S_{i-1, n-(i-1)}^{l, k-l}, S_{i+1, n-(i+1)}^{l, k-l}, S_{i, n-1}^{l-1, k-(l-1)}$ or $S_{i, n-1}^{l+1, k-(l+1)}$ in moment $(t+\Delta t)$ will happen in state $S_{i, n-i}^{l, k-l}$, \{quantity of devices: operable- $i$, inoperable $-(n-i)$; quantity of requests: general quantity- $k, l$-in service, $(k-l)$ in queue for service $\}$. In the given case it is supposed that transitions between system states are realized due to request income and their service at fixed values of lower indices $i$ and $(n-i)$ (i.e. parameters of refusals and repairs of service devices);

$P_{i, n-i}^{l, k-l}(t+\Delta t)$ is the probability of the event when the system being in time moment $t$ in one of the possible states $S_{i, n-i}^{l, k-l}, \quad S_{i, n-1}^{l-1, k-(l-1)}$ or $S_{i, n-1}^{l+1, k-(l+1)}$ in moment $(t+\Delta t)$ will happen in state $S_{i, n-i}^{l, k-l}$, \{quantity of devices: operable - $i$, inoperable - $(n-i)$; quantity of requests: general quantity $-k, l$-in service, $(k-l)$ in queue for service . In the given case it is supposed that transitions between system states are realized due to refusals and repairs of service devices at fixed values of upper indices $l$ and $k$ (i.e. parameters of request income and their service);

$\left(1-a_{i, n-i}^{l, k-l} \Delta t\right) R_{i, n-i}^{l, k-l}(t)$ is the probability of the event when the system with probability $R_{i, n-i}^{l, k-l}(t)$ being in moment $t$ in state $S_{i, n-i}^{l, k-l}(t)$ \{quantity of devices: operable-i, inoperable- $(n-i)$; quantity of requests: general quantity $-k, l$-in service, $(k-l)$-in queue for service $\}$ in time $\Delta t$ with probability $\left(1-a_{i, n-i}^{l, k-l} \Delta t\right)$ will not go over to neither of possible states and will stay in the former state;

$a_{i-1, n-(i-1)}^{l, k-l} R_{i-1, n-(i-1)}^{l, k-l}(t) \Delta t$ is the probability of the event when the system with probability $R_{i-1, n-(i-1)}^{l, k-l}(t)$ being in moment $t$ in state $S_{i-1, n-(i-1)}^{l, k-l}(t)$ \{quantity of devices: operable- $(i-1)$, inoperable- $[n-(i-1)]$; quantity of 
requests: general quantity $-k, l$-in service, $(k-l)$ in queue for service $\}$ in time $\Delta t$ with probability $a_{i-1, n-(i-1)}^{l, k-1} \Delta t$ will not go over to state $R_{i, n-i}^{l, k-l}(t)$;

$a_{i+1, n-(i+1)}^{l, k-l} R_{i+1, n-(i+1)}^{l, k-1}(t) \Delta t$ is the probability of the event when the system with probability $R_{i+1, n-(i+1)}^{l, k-l}(t)$ being in moment $t$ in state $S_{i+1, n-(i+1)}^{l, k-1}(t)$ \{quantity of devices: operable $-i+1$, inoperable- $[n-(i+1)]$; quantity of requests: general quantity-k, $l$-in service, $(k-l)$ in queue for service) in time $\Delta t$ with probability $a_{i-1, n-(i-1)}^{l, k-l} \Delta t \quad$ will go over to state $S_{i, n-i}^{l, k-l}(t)$;

$\left(1-b_{i, n-i}^{l, k-l} \Delta t\right) P_{i, n-i}^{l, k-l}(t)$ is the probability of the event when the system with probability $P_{i, n-i}^{l, k-l}(t)$ being in moment $t$ in state $S_{i, n-i}^{l, k-l}(t)$ \{quantity of devices: operable $-i$, inoperable $-(n-i)$; quantity of requests: general quantity — $k$, $l$-in service, $(k-l)$ in queue for service) in time $\Delta t$ with probability $\left(1-b_{i, n-i}^{l, k-l}\right) \Delta t$ will not go over to neither of possible states and will stay in the former state;

$b_{i, n-i}^{l-1, k-(l-1)} P_{i, n-i}^{l-1, k-(l-1)}(t) \Delta t$ is the probability of the event when the system with probability $P_{i, n-i}^{l-1, k-(l-1)}(t)$ being in moment $t$ in state $S_{i, n-i}^{l-1, k-(l-1)}(t)$ \{quantity of devices: operable- $i$, inoperable- $(n-i)$; quantity of requests: general quantity-k, $(l-1)$-in service, $[k-(l-1)]$ in queue for service $\}$ in time $\Delta t$ with probability $b_{i, n-i}^{l-1, k-(l-1)} \Delta t$ will go over to state $S_{i, n-i}^{l, k-l}(t)$;

$b_{i, n-i}^{l+1, k-(l+1)} P_{i, n-i}^{l+1, k-(l+1)}(t) \Delta t$ is the probability of the event when the system with probability $P_{i, n-1}^{l+1, k-(l+1)}(t)$ being in moment $t$ in state $S_{i, n-i}^{l+1, k-(l+1)}(t)$ \{quantity of devices: operable- $i$, inoperable- $(n-i)$; quantity of requests: general quantity $-k, l+1$-in service, $[k-(l+1)]$ in queue for service) in time $\Delta t$ with probability $b_{i, n-i}^{l+1, k-(l+1)} \Delta t$ will go over to state $P_{i, n-i}^{l, k-l}(t)$.

From Equations (1), (2) and (3) by going to limit at $\Delta t \rightarrow 0$ we obtain the following recurrence differential equations:

$$
\begin{gathered}
\frac{\mathrm{d} R_{0, n}^{l, k-l}(t)}{\mathrm{d} t}=a_{0, n}^{l, k-l} R_{0, n}^{l, k-l}(t)+a_{1, n-1}^{l, k-l} R_{1, n-1}^{l, k-l}(t)+Q_{0, n-1}^{l, k-l}(t) ; \quad \text { when } i=0 ; \\
\frac{\mathrm{d} R_{i, n-i}^{l, k-l}(t)}{\mathrm{d} t}=a_{i, n-i}^{l, k-l} R_{i, n-i}^{l, k-l}(t)+a_{i-1, n-(i-1)}^{l, k-l} R_{i-1, n-(i-1)}^{l, k-1}(t)+a_{i+1, n-(i+1)}^{l, k-l} R_{i+1, n-(i+1)}^{l, k-l}(t)+Q_{i, n-1}^{l, k-l}(t) ; \quad \text { when } 1 \leq i \leq n ;
\end{gathered}
$$

where

$$
\begin{gathered}
Q_{i, n-1}^{l, k-l}(t)=b_{i, n-i}^{l-1, k-(l-1)} P_{i, n-1}^{l-1, k-(l-1)}(t)+b_{i, n-i}^{l+1, k-(l+1)} P_{i, n-1}^{l+1, k-(l+1)}(t) \\
\frac{\mathrm{d} P_{i, n-i}^{0, k}(t)}{\mathrm{d} t}=b_{i, n-i}^{0, k} P_{i, n-i}^{0, k}(t)+b_{i, n-i}^{1, k-1} P_{i, n-1}^{1, k-1}(t) \text {, when } l=0 ;
\end{gathered}
$$

$$
\frac{\mathrm{d} P_{i, n-i}^{l, k-l}(t)}{\mathrm{d} t}=b_{i, n-i}^{l, k-l} P_{i, n-i}^{l, k-l}(t)+b_{i, n-i}^{l-1, k-(l-1)} P_{i, n-1}^{l-1, k-(l-1)}(t)+b_{i, n-i}^{l+1, k-(l+1)} P_{i, n-1}^{l+1, k-(l+1)}(t) ; \quad \text { when } 1 \leq l \leq k
$$

Here: $\frac{\mathrm{d} R_{i, n-i}^{l, k-l}(t)}{\mathrm{d} t}=\lim _{\Delta t \rightarrow 0} \frac{R_{i, n-i}^{l, k-l}(t+\Delta t)-R_{i, n-i}^{l, k-l}(t)}{\Delta t}, \frac{\mathrm{d} P_{i, n-i}^{l, k-l}(t)}{\mathrm{d} t}=\lim _{\Delta t \rightarrow 0} \frac{P_{i, n-i}^{l, k-l}(t+\Delta t)-P_{i, n-i}^{l, k-l}(t)}{\Delta t}$.

Systems of Equations (6), (7), (9) and (10) represent the systems of inhomogeneous differential equations with fixed factors.

In assumption of given starting conditions:

$R_{i, n-i}^{l, k-l}(0)=R_{n, 0}^{0, k}(0)=1, \quad P_{i, n-i}^{l, k-l}(0)=P_{n, 0}^{0, k}(0)=1$ partial solutions of these equations can be retrieved by method of operational calculus using the following Laplace transformations: 


$$
\begin{gathered}
L\left\{\frac{\mathrm{d} R_{i, n-i}^{l, k-l}(t)}{\mathrm{d} t}\right\}=s \bar{R}_{i, n-i}^{l, k-l}(s)-R_{n, 0}^{0, k}(0)=s \bar{R}_{i, n-i}^{l, k-l}(s)-1 / s ; \quad L\left\{R_{i, n-i}^{l, k-l}(t)\right\}=s \bar{R}_{i, n-i}^{l, k-l}(s) ; \\
L\left\{R_{i-1, n-(i-1)}^{l, k-l}(t)\right\}=\bar{R}_{i-1, n-(i-1)}^{l, k-l}(s) ; \quad L\left\{R_{i+1, n-(i+1)}^{l, k-l}(t)\right\}=\bar{R}_{i+1, n-(i+1)}^{l, k-l}(s) ; \quad L\left\{Q_{i, n-1}^{l, k-l}(t)\right\}=\bar{Q}_{i, n-1}^{l, k-l}(s) . \\
L\left\{\frac{\mathrm{d} P_{i, n-i}^{l, k-l}(t)}{\mathrm{d} t}\right\}=s \bar{P}_{i, n-i}^{l, k-l}(s)-P_{n, 0}^{0, k}(t)=s \bar{P}_{i, n-i}^{l, k-l}(s)-1 / s ; \quad L\left\{P_{i, n-i}^{l, k-l}(t)\right\}=\bar{P}_{i, n-i}^{l, k-l}(s) ; \\
L\left\{P_{i, n-1}^{l-1, k-(l-1)}(t)\right\}=\bar{P}_{i, n-1}^{l-1, k-(l-1)}(s) ; \quad L\left\{P_{i, n-1}^{l+1, k-(l+1)}(t)\right\}=\bar{P}_{i, n-1}^{l+1, k-(l+1)}(s) ; \quad L\{1\}=1 / s .
\end{gathered}
$$

Systems of Equations (6)-(10) in Laplace derivatives will get the following form:

$$
\begin{aligned}
s \bar{R}_{0, n}^{l, k-l}(s)-1 / s= & a_{0, n}^{l, k-l} \bar{R}_{0, n}^{l, k-l}(s)+a_{1, n-1}^{l, k-l} \bar{R}_{1, n-1}^{l, k-l}(s)+\bar{Q}_{0, n-1}^{l, k-l}(s) ; \text { when } i=0 ; \\
s \bar{R}_{i, n-i}^{l, k-l}(s)-\frac{1}{s}= & a_{i, n-i}^{l, k-l} \bar{R}_{i, n-i}^{l, k-l}(s)+a_{i-1, n-(i-1)}^{l, k-1} \bar{R}_{i-1, n-(i-1)}^{l, k-l}(s) \\
& +a_{i+1, n-(i+1)}^{l, k-l} \bar{R}_{i+1, n-(i+1)}^{l, k-l}(s)+\bar{Q}_{i, n-1}^{l, k-l}(s), \quad \text { when } 1 \leq i \leq n ;
\end{aligned}
$$

where

$$
\begin{gathered}
\bar{Q}_{i, n-1}^{l, k-l}(s)=b_{i, n-i}^{l-1, k-(l-1)} \bar{P}_{i, n-1}^{l-1, k-(l-1)}(s)+b_{i, n-i}^{l+1, k-(l+1)} \bar{P}_{i, n-1}^{l+1, k-(l+1)}(s) ; \\
s \bar{P}_{i, n-i}^{0, k}(s)-1 / s=b_{i, n-i}^{0, k} \bar{P}_{i, n-i}^{0, k}(s)+b_{i, n-i}^{1, k-1} \bar{P}_{i, n-1}^{1, k-1}(s) \text {, when } l=0 ; \\
s \bar{P}_{i, n-i}^{l, k-l}(s)-1 / s=b_{i, n-i}^{l, k-l} \bar{P}_{i, n-i}^{l, k-l}(s)+b_{i, n-i}^{l-1, k-(l-1)} \bar{P}_{i, n-1}^{l-1, k-(l-1)}(s)+b_{i, n-i}^{l+1, k-(l+1)} \bar{P}_{i, n-1}^{l+1, k-(l+1)}(s) \text {, when } 1 \leq l \leq k
\end{gathered}
$$

After simple transformations, Equations (11)-(15) are transformed into inhomogeneous systems of algebraic equations which are solved quite simply with Cramer formulas relative to $\bar{R}_{i, n-i}^{l, k-l}(s)$ и $\bar{P}_{i, n-i}^{l, k-l}(s)$ :

$$
\begin{gathered}
\left(s-a_{0, n}^{l, k-l}\right) \bar{R}_{0, n}^{l, k-l}(s)-a_{1, n-1}^{l, k-1} \bar{R}_{1, n-1}^{l, k-l}(s)=\frac{\left[1+s \bar{Q}_{0, n-1}^{l, k-l}(s)\right]}{s} \text {, when } i=0 ; \\
\left(s-a_{i, n-i}^{l, k-l}\right) \bar{R}_{i, n-i}^{l, k-l}(s)-a_{i-1, n-(i-1)}^{l, k-l} \bar{R}_{i-1, n-(i-1)}^{l, k}(s)-a_{i+1, n-(i+1)}^{l, k-1} \bar{R}_{i+1, n-(i+1)}^{l, k-l}(s)=\frac{\left[1+s \bar{Q}_{i, n-1}^{l, k-l}(s)\right]}{s}, \quad \text { when } 1 \leq i \leq n ;
\end{gathered}
$$

where

$$
\begin{gathered}
\bar{Q}_{i, n-1}^{l, k-l}(s)=b_{i, n-i}^{l-1, k-(l-1)} \bar{P}_{i, n-1}^{l-1, k-(l-1)}(s)+b_{i, n-i}^{l+1, k-(l+1)} \bar{P}_{i, n-1}^{l+1, k-(l+1)}(s) ; \\
\left(s-b_{i, n-i}^{0, k}\right) \bar{P}_{i, n-i}^{0, k}(s)-b_{i, n-i}^{1, k-1} \bar{P}_{i, n-i}^{1, k-1}(s)=1 / s \text {, when } l=0 ; \\
\left(s-b_{i, n-i}^{l, k-l}\right) \bar{P}_{i, n-i}^{l, k-l}(s)+b_{i, n-i}^{l-1, k-(l-1)} \bar{P}_{i, n-1}^{l-1, k-(l-1)}(s)+b_{i, n-i}^{l+1, k-(l+1)} \bar{P}_{i, n-1}^{l+1, k-(l+1)}(s)=1 / s ; \quad \text { when } 1 \leq i \leq n
\end{gathered}
$$

With the help of Cramer formula the solution of Equation (20) can be written in the following form:

$$
\bar{P}_{i, n-i}^{l, k-l}(s)=\bar{\Delta}_{\delta}^{l, k-l}(s) / \bar{\Delta}^{l, k-l}(s) \text {, при } 1 \leq i \leq n,
$$

where

$$
\bar{\Delta}^{l, k-1}(s)=\left[\begin{array}{cccc}
{\left[s-\bar{b}_{i, n-i}^{1, k-1}(s)\right]} & \bar{b}_{i, n-1}^{0, k-0}(s) & \cdots & \bar{b}_{i, n-i}^{2, k-2}(s) \\
\vdots & \vdots & & \vdots \\
{\left[s-\bar{b}_{i, n-i}^{k-1,1}(s)\right]} & \bar{b}_{i, n-i}^{k-2,2}(s) & \cdots & \bar{b}_{i, n-i}^{k, 0}(s)
\end{array}\right]
$$

is a principal determinant of system (20), while $\bar{\Delta}_{\delta}^{l, k-l}(s)$ are determinants received by substitution of the right part (1/s) of equation system (20) into $\delta$ th column of matrix. Here $\delta=1-3$ are the numbers of matrix columns. 
The solutions of the system of Equations (17) are written similarly:

$$
\bar{R}_{i, n-i}(s)=\bar{\Delta}_{i, n-i}^{\delta}(s) / \bar{\Delta}^{l, k-l}(s), \quad \text { when } 1 \leq i \leq n,
$$

where

$$
\bar{\Delta}_{i, n-i}(s)=\left[\begin{array}{cccc}
{\left[s-\bar{a}_{i, n-i}^{1, k-1}(s)\right]} & \bar{a}_{i, n-1}^{0, k-0}(s) & \cdots & \bar{a}_{i, n-i}^{2, k-2}(s) \\
\vdots & \vdots & & \vdots \\
{\left[s-\bar{a}_{i, n-i}^{k-1,1}(s)\right]} & \bar{a}_{i, n-i}^{k-2,2}(s) & \cdots & \bar{a}_{i, n-i}^{k, 0}(s)
\end{array}\right]
$$

is a principal determinant of system (17), while $\bar{\Delta}_{i, n-i}^{\delta}(s)$ are determinants received by substitution of the right part $\left(-\left[1+s \bar{Q}_{i, n-1}^{l, k-l}(s)\right] / s\right)$ of equation system (12) into $\delta$-th column of matrix. Here, as above $\delta=1-3$ are the numbers of matrix columns.

With the help of found probabilities of states it is easy to determine different factors of dependability and efficiency of the examined system.

\section{Conclusions}

The results received in the given work represent some generalization of the results already known in literature. They enable to calculate probabilities of possible states of above described system with which other important factors of dependability and efficiency of functioning of such systems can be expressed.

The results of the work can be successfully used in analysis and synthesis of such complex technical systems as are data transfer systems, cellular communication systems, computer and communication networks, flexible manufacturing lines, automated control systems and also other complex technical systems used in civil as well as in military purposes. They can be used for estimation of preset or provision of required levels of dependability, productivity and effectiveness of functioning of complex technical systems on the stage of exploitation, as well as design.

\section{References}

[1] Gnedenko, B.V. and Kovalenko, I.N. (2012) Introduction to Queuing Theory. LKT, 400.

[2] Saati, T.L. (1965) Elements of Queuing Theory and Its Application. Sovetskoe Radio, 510.

[3] Cherkesov, G.N. (1974) Dependability of Technical Systems with Time Redundancy. Sovetskoe Radio, 296.

[4] Feller, W. (1971) An Introduction to Probability Theory and Its Applications. Vol. 2, John Willey and Sons, New York, 766.

[5] Mikadze, Z.I., Mikadze, I.S. and Khocholava, V.V. (2007) On One Multichannel Mixed Queuing System with Limited Waiting Time. Automatics and Telemechanics, 7, 44-51.

[6] Khocholava, V.V. and Mikadze, I.S. (2002) On One Model of Queuing System. Georgian Engineering News, 4, 47-52.

[7] Khurodze, R.A., Khocholava, V.V. and Mikadze, I.S. (2003) On One Queuing System with Unreliable Queuing System. Problems of Applied Mechanics, 3, 9-18.

[8] Mikadze, I.S. and Khocholava, V.V. (2004) On One Model of Information Transition by Unreliable Communication Channel. Automatics and Telemechanics, 8, 85-90.

[9] Mikadze, I.S., Khocholava, V.V. and Khurodze, R.A. (2004) Virtual Waiting Time in Single-Line QS with Unreliable Devices. Automatics and Telemechanics, 12, 119-128.

[10] Khurodze, R.A. and Kakubava, R.V. (2005) Some Mathematical Aspects of Dependability Planning of Technical Systems. Bulletin of the Georgian Academy of Sciences, 171, 449-452.

[11] Khurodze, R.A., Shamugia, R.R. and Kukava, R.R. (2006) Research of Dependability Characteristics of Technical Systems with Equipment and Time Duplications. Bulletin of the Georgian National Academy of Sciences, 174, 110113. 
Scientific Research Publishing (SCIRP) is one of the largest Open Access journal publishers. It is currently publishing more than 200 open access, online, peer-reviewed journals covering a wide range of academic disciplines. SCIRP serves the worldwide academic communities and contributes to the progress and application of science with its publication.

Other selected journals from SCIRP are listed as below. Submit your manuscript to us via either submit@scirp.org or Online Submission Portal.
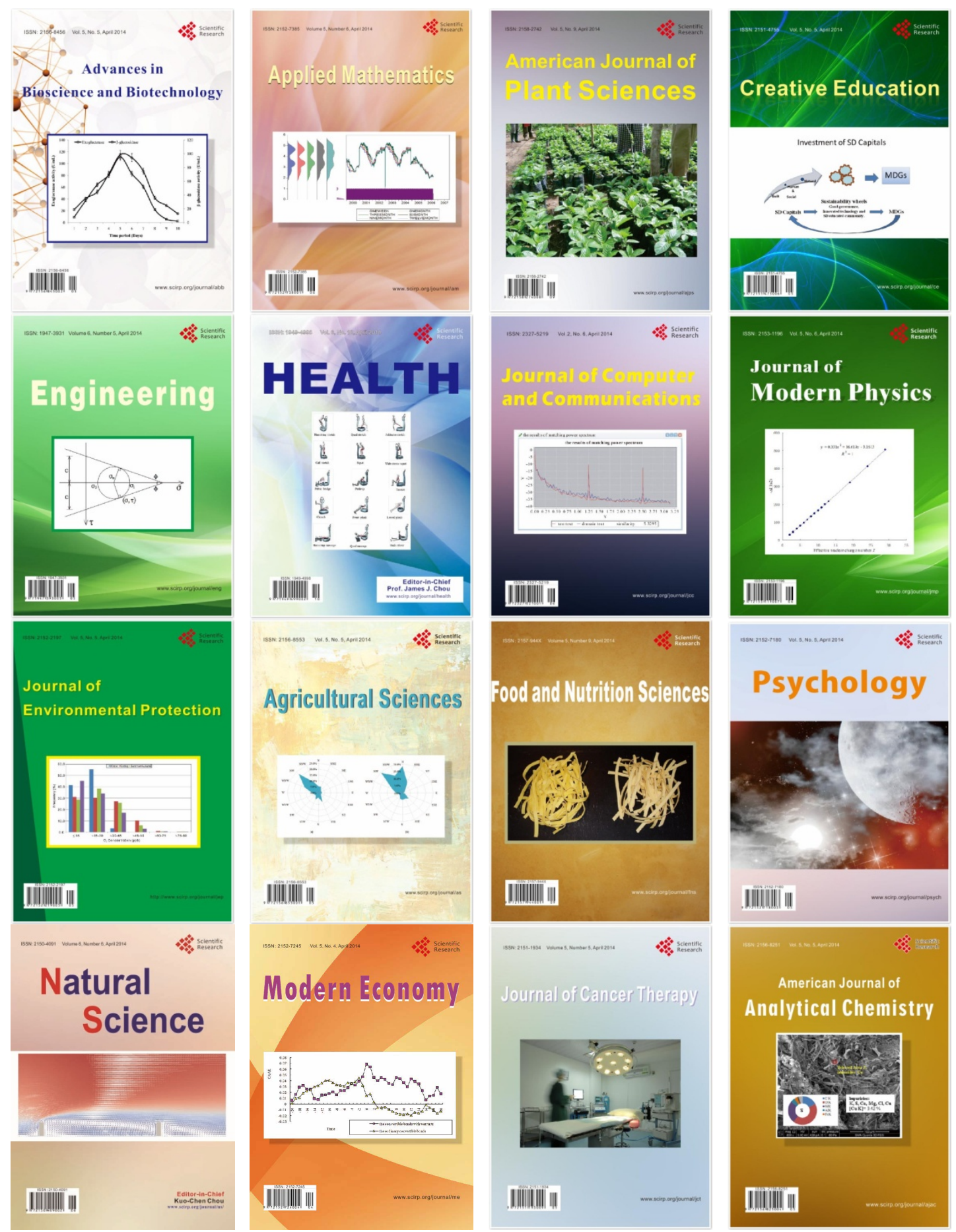